\title{
Datos actuales sobre el sistema de cocción de clínker I.H.I. - S.F.
}

T. KOBAYASHI

Ishikawajima-Harima Heavy Industries Co. Ltd.

(Tokyo)

Felguera I.H.I., S. A.

(España)

\section{N T R R O D U C C I O N}

En este artículo tenemos la ocasión de informar acerca de una nueva técnica en el campo de la producción de clínker de cemento,que ha sido desarrollada por IshikawajimaHarima Heavy Industries y es conocida como el sistema I.H.I.-S.F.

Desde el año 1963 las investigaciones de I.H.I. han sido enfocadas hacia la sustitución del horno rotativo, de baja productividad, por uno de tipo estático, pequeño tamaño y gran eficiencia. En 1968 se aplicó por primera vez en sistema I.H.I.-S.F. en una planta de calcinación de alúmina de las industrias Químicas Sumitomo, tras varias pruebas realizadas con éxito en los laboratorios. Desde entonces se han instalado 5 más, con una producción entre las 500 y 800 t/año de alúmina.

Se prosiguió en las investigaciones con el fin de aplicar este concepto a la industria del cemento; así, en el año 1971 empezó a funcionar una planta de 2.000 t/día de clínker de cemento en la Cementera Chichibu n. ${ }^{\circ}$, con el sistema S.F.

Con el proceso S.F., más del $60 \%$ del fuel-oil se quema en el horno estático instantáneo (horno flash), realizándose la reacción de calcinación en la harina cruda en un nivel aproximado del $90 \%$.

Así pues, la mitad del trabajo que realizaría un horno rotativo con un sistema seco convencional podría ser realizado por un horno estático.

P.or ello I.H.I. es reconocida como la primera empresa del mundo que ha desarrollado tal sistema de precalcinación, en el sentido más estricto de la palabra.

La figura 1 muestra la más reciente evolución en Japón de los sistemas de producción de clínker, según el informe de la Asociación Industrial del Cemento.

Hace veinticinco años, una compañía alemana iritrodujo un sistema de producción de clínker caracterizado por un horno rotativo y un intercambiador de suspensión: era el sistema S.P. En aquel momento se había desarrollado multitud de nuevos experimentos y este sistema alcanzó la primera posición entre todos ellos.

Debido a los esfuerzos de las industrias de cemento para incrementar la producción y reducir los gastos de construcción, costo y mantenimiento, se han encontrado límites técnicos al sistema S.P. 
Por esta razón, la industria cementera japonesa ha aceptado rápidamente el sistema de precalcinación, ocupando éste ahora el primer puesto en cuanto a sistemas de producción, por encima incluso del S.P. Como primer constructor, I.H.I. ha realizado más del $60 \%$ de las plantas con el nuevo sistema I.H.I.-S.F. de precalcinación.

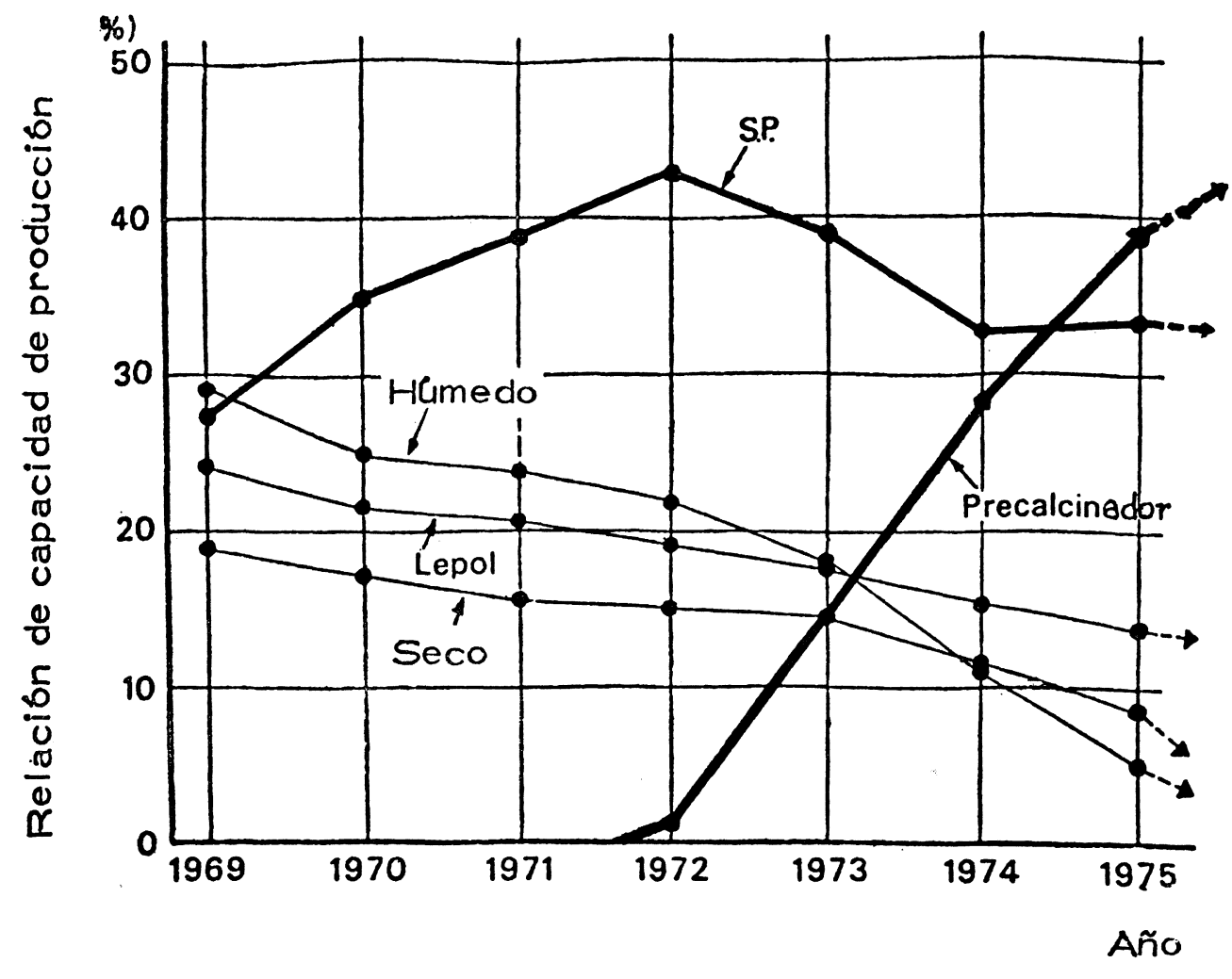

Fig. 1.-Porcentaje de producción en varios sistemas.

\section{DESCRIPCION DEL SISTEMA S.F.}

El sistema I.H.I.-S.F. se puede explicar fácilmente siguiendo el diagrama de flujo de la figura 2.

La planta de clínkerización está compuesta (siguiendo el flujo de material) de un precalentador de suspensión, similar a los del tipo convencional; un horno de calcinación -el horno flash- desarrollado por I.H.I.; un horno rotativo y un enfriador de clínker.

Los principios de este proceso son los siguientes:

1. Una reacción de calcinación, endotérmica y que requiere más de la mitad de las calorías necesarias en todo el proceso de clínkerización. Esta reacción tiene lugar en el horno flash. La calcinación se realiza en cuestión de segundos, debido al alto rendimiento en intercambio de calor que tiene lugar en el horno flash.

2. Reacción de sinterización, exotérmica; se realiza en un horno rotativo relativamente pequeño, con un adecuado tiempo de retención, usando el calor irradiado por los gases a alta temperatura. 
El diagrama esquemático (fig. 3) muestra las diferencias entre los sistemas S.F. y S.P. aún más claramente.

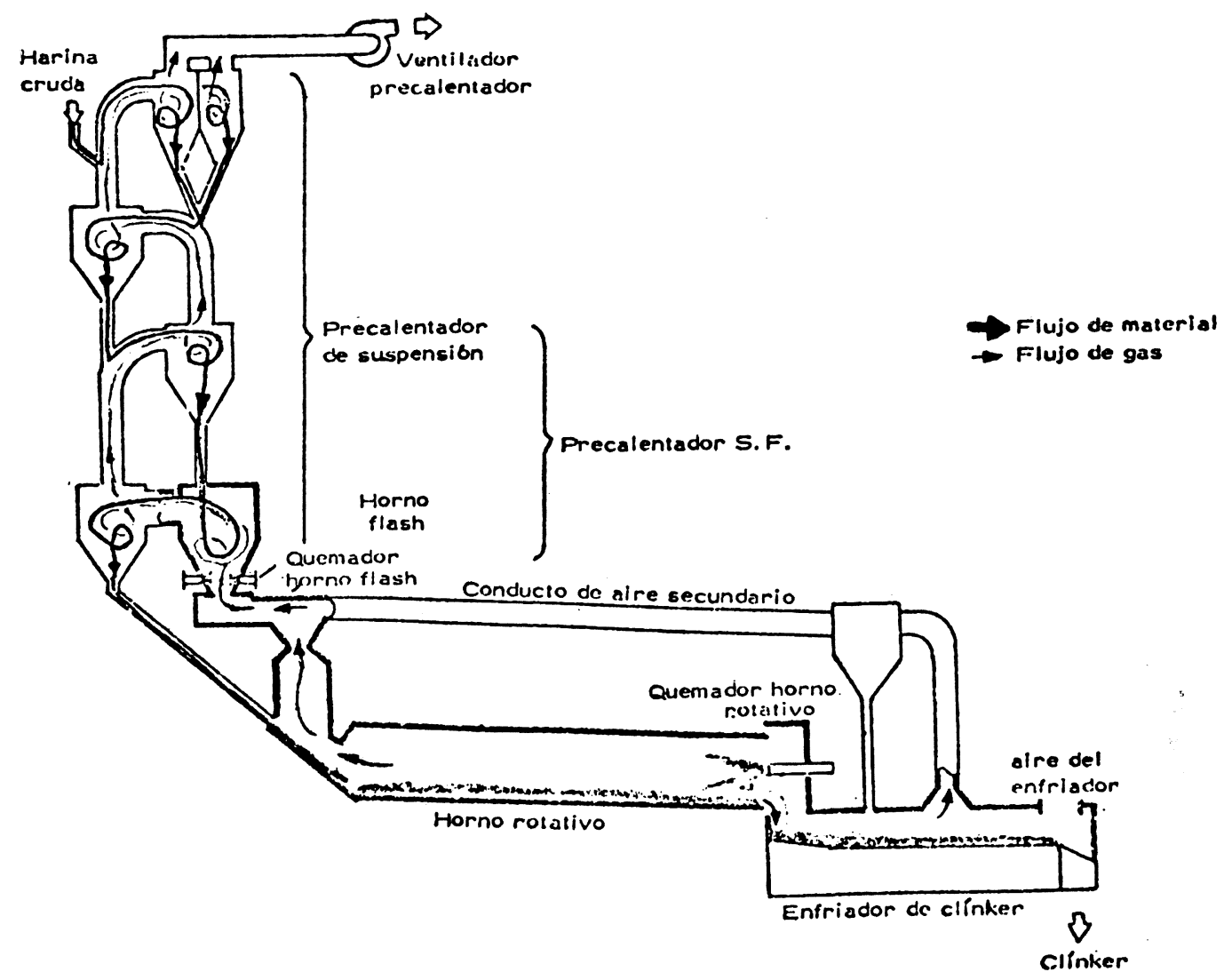

Fig. 2.-Esquema de flujo en el sistema S.F.

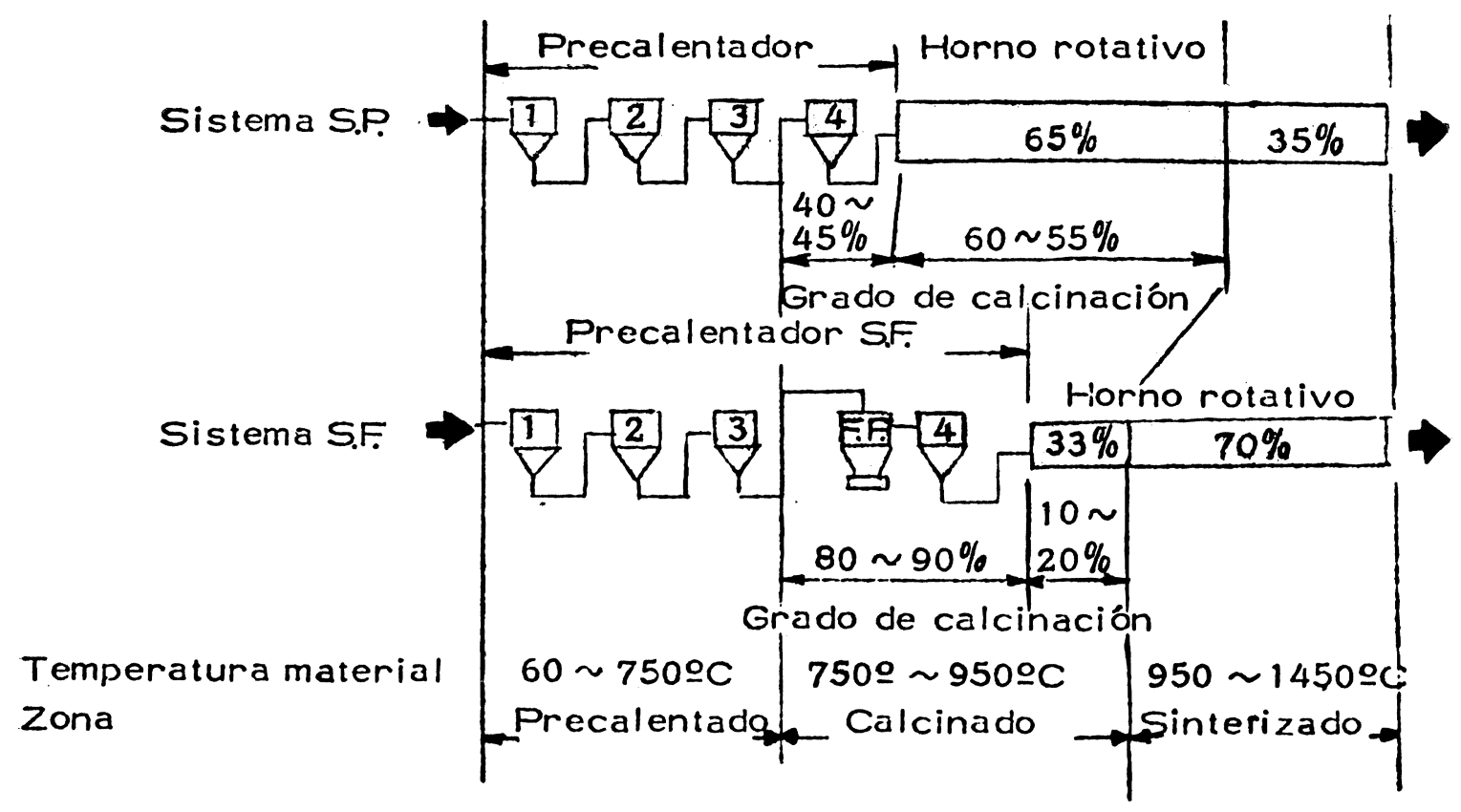

Fig. 3.-Comparación entre el S.P. y el S.F. 
Con el sistema S.P., en el precalentador de suspensión se obtiene como máximo un $45 \%$ de calcinación sobre el total, efectuándose el resto en el horno rotativo. Así pues, casi las dos terceras partes del volumen del horno rotativo han de ser utilizadas para la reacción de calcinación.

No hay duda de que el horno rotativo es un intercambiador de calor razonable, a lo largo del cual es transferido el calor, principalmente por radiación, igual que en la zona de sinterización. Sin embargo, la transferencia de calor en la zona de menor temperatura del horno tiene un rendimiento muy bajo comparándolo con otros tipos de intercambiadores, en los que los gases están en contacto con las partículas directamente.

Los esfuerzos por lograr un incremento de calcinación en el precalentador están limitados por la presencia de elementos volátiles, tales como los álcalis, cloro y azufre, los cuales se evaporan en el horno rotativo, circulando a lo largo de todo el proceso.

En otras palabras, si el calor transferido en el horno rotativo se pudiese trasladar al precalentador S.P., con el fin de realizar en él una mayor calcinación, la temperatura de los gases que saliesen del horno hacia el precalentador deberían estar por encima de los $1.200^{\circ} \mathrm{C}$. Consecuentemente, realizar esto en un proceso convencional no es posible, porque aparecerían las sustancias volátiles antes mencionadas en el precalentador. El nuevo sistema S.F. ha resuelto este problema por medio del horno flash, en el cual la combustión y la transferencia de calor a las partículas de harina cruda tienen lugar simultáneamente.

El horno flash ha conseguido producir alrededor del $90 \%$ de calcinación de la harina cruda a la entrada del horno rotativo. Así pues, las calorías a producir en el horno rotativo son reducidas a la mitad frente al sistema convencional S.P., y, como consecuencia, el rendimiento volumétrico se duplica.

Aquí se explica en detalle la estructura y operación de un horno flash, según puede apreciarse en el dibujo (fig. 4).

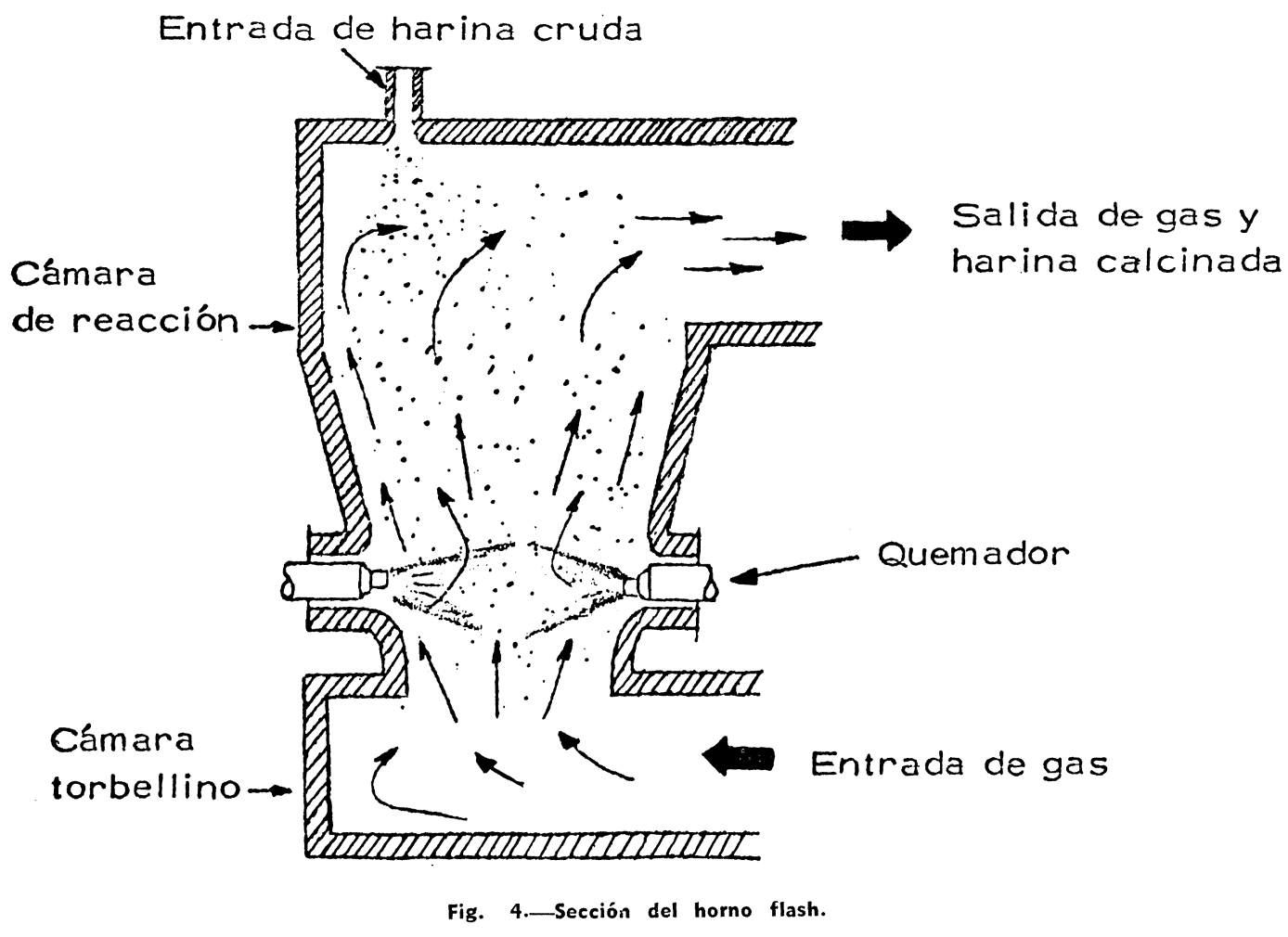


El recipiente del horno consiste básicamente en una cámara de reacción (parte superior) y una cámara torbellino (parte inferior).

La entrada de harina cruda está situada en el techo de la cámara de reacción y los quemadores (en número variable) en la parte más inferior de esta cámara.

Las partículas de harina cruda precalentadas son introducidas en la cámara de reacción de arriba hacia abajo, mientras el fuel-oil atomizado es inyectado horizontalmente. A través de la cámara de torbellino se induce una mezcla de gases que provienen del horno rotativo y aire de combustión precalentado; estos gases entran en la cámara de reacción con una gran turbulencia.

La figura 5 muestra el modelo de flujo en el horno flash. La mayor parte de los gases asciende de la cámara torbellino a lo largo de las paredes de la cámara de reacción afectada de una gran turbulencia junto con partículas de harina cruda en suspensión. Esta mezcla de gases y partículas completa una o más revoluciones, subiendo hacia el conducto de salida de gases. Debido al remolino creado, se crea una zona de presiones negativas a lo largo del eje axil de la cámara de reacción. La harina cruda y el fuel-oil son introducidos en esta zona de depresión. De este modo se mezclan con los gases. Cuando las partículas de fuel se queman, el calor desarrollado se transmite a las partículas de harina cruda, instantáneamente. Por eso, la luminosidad de la llama de combustión que se observa en el horno rotativo no es observada en el horno flash.

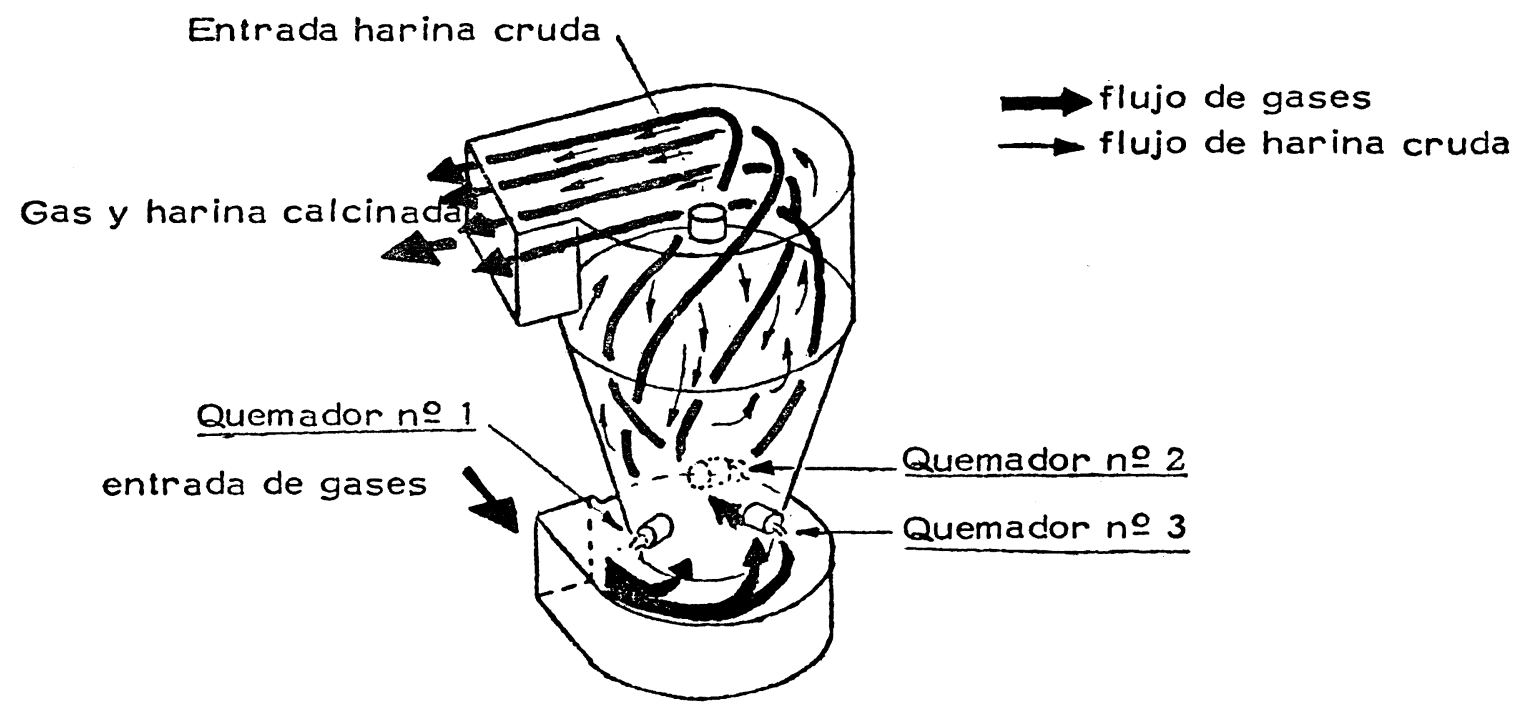

Fig. 5.-Diagrama de flujos en el horno flash.

De hecho, alrededor del $90 \%$ del calor producido en el horno flash es transferido por convección forzada, siéndolo sólo en un $10 \%$ por radiación.

Desde el punto de vista del principio de operación explicado, no cabe duda de que el horno flash por sí mismo tiene excelentes posibilidades de conseguir el intercambio de calor en un pequeño espacio. Cuando esto se aplica al proceso de clínkerización de cemento, es imprescindible que la harina cruda y las partículas de fuel estén uniformemente distribuidas y que, como resultado, la temperatura de salida de gas en el horno sea mantenida uniforme. Esto proviene de las materias volátiles que normalmente contienen la harina cruda y el fuel, tales como álcalis, cloro y azufre (en estado vaporizado debido al precalentamiento). 
El modelo actual del horno flash, la posición de los quemadores, la forma de alimentar la harina y otros detalles, han sido definidos después de realizar repetidas mediciones de temperaturas y observaciones de las líneas de flujo. En la figura 6 se muestra un ejemplo de la distribución de temperaturas en el horno flash tomadas en tres secciones diferentes. Según la zona del horno, la temperatura oscila entre los $830^{\circ}$ y $900^{\circ} \mathrm{C}$.

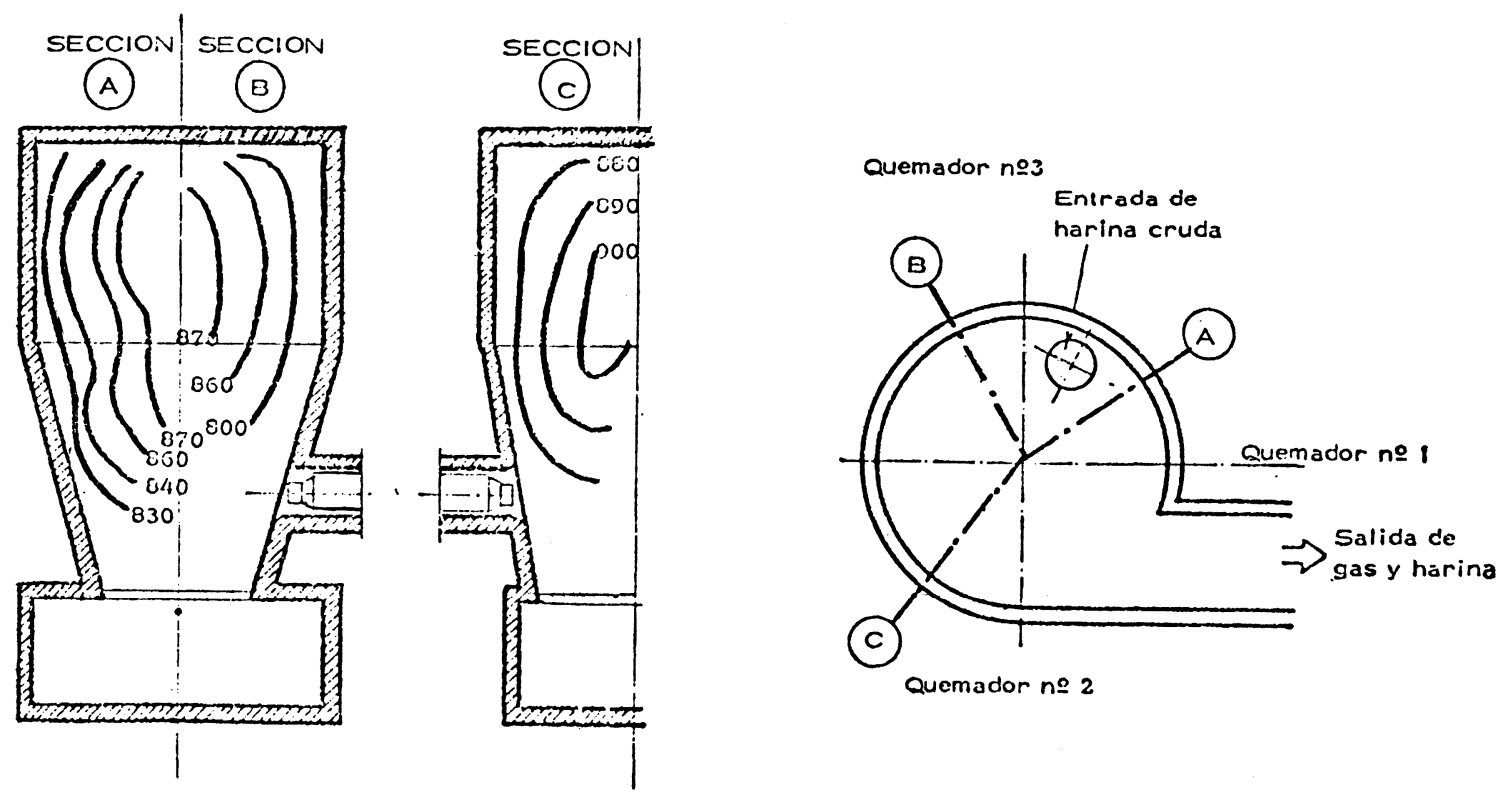

Fig. 6.-Distribución de temperaturas en el horno flash $\left({ }^{\circ} \mathrm{C}\right)$.

\section{OPERACION Y PROCESO}

La estructura y operación del proceso como todo el sistema es explicado en detalle. Como un ejemplo, se muestran en la tabla 1 las especificaciones de la mayor planta del mundo de producción de cemento, precisamente con el sistema S.F. Se trata de la que Cementos Chichibu tiene instalada en Kumagaya (Japón).

Debido a esta gran producción, el precalentador S.F. consiste en dos unidades paralelas, cada una de las cuales posee cuatro etapas de ciclones, por lo que se le llama tipo doble. El horno flash de cada unidad está situado entre la tercera y la cuarta etapa siguiendo el fluja del material, y poseen cuatro quemadores cada uno. El horno rotativo tiene $5,5 \mathrm{~m}$ de diámetro y $100 \mathrm{~m}$ de longitud y va equipado con un quemador Pillard incorporando 3 boquillas en una lanza. El enfriador de clínker es un Fuller de tres etapas tipo parrilla con una puerta de venteo para enviar aire de combustión caliente al horno flash.

El gas sucio del precalentador se hace pasar a través de un secador giratorio para obtener un secado de la arcilla y por tres molinos para materiales crudos; de aquí pasa a un electrofiltro desarrollado así mismo por I.H.I.

El aire de venteo del enfriador de clínker contiene de 10 a $30 \mathrm{~g} / \mathrm{m}^{3}$ normal de polvo de clínker, el cual es limpiado por una combinación de multiciclones y filtro electrostático. La concentración de polvo en los gases limpios procedentes de todos los electrofiltros es menor de $30 \mathrm{mg} / \mathrm{m}^{3}$ normal. 
TABLA 1

Especificaciones de equipos para una planta S.F. de 8.000 t/día

\begin{tabular}{|c|c|c|c|c|c|}
\hline Equipo & Especir & ficación principal & Equipo & Especi & ficación principal \\
\hline $\begin{array}{l}\text { Precalenta- } \\
\text { dor S.F. }\end{array}$ & $\begin{array}{l}\text { Tipo } \\
\text { Modelo } \\
\text { Horno flash } \\
\text { Quemador } \\
\text { Ventilador tiro } \\
\text { inducido } \\
\text { Accionamiento } \\
\text { ventilador } \\
\text { Torre }\end{array}$ & $\begin{array}{l}\text { I.H.I.-S.F. cuatro etapas } \\
237 \\
2-7,4 \mathrm{~m} \varnothing, 280 \mathrm{dm}^{3} \\
\text { 2-Máx. } 9 \mathrm{t} / \mathrm{h} \\
2-9.700 \mathrm{~m}^{3} / \mathrm{min}, 350^{\circ} \mathrm{C} \\
770 \mathrm{~mm} \mathrm{columna} \mathrm{de} \\
\text { agua } \\
2-2.100 \mathrm{~kW} \\
24 \mathrm{~m} \mathrm{~A} \times 17 \mathrm{~m} \mathrm{~L} \times \\
\times 68,6 \mathrm{~m} \mathrm{H}\end{array}$ & $\begin{array}{l}\text { Enfriador de } \\
\text { clínker }\end{array}$ & $\begin{array}{l}\text { Tipo } \\
\text { Modelo } \\
\text { Area de parri- } \\
\text { llas } \\
\text { Velocidad de } \\
\text { parrillas } \\
\text { Accionamiento } \\
\text { N. de compar- } \\
\text { timen to de } \\
\text { aire de en- } \\
\text { friamiento } \\
\text { N. }{ }^{\circ} \text { ventilado- } \\
\text { res }\end{array}$ & $\begin{array}{l}\text { Fuller de tres etapas } \\
1.430 \mathrm{~S} / 1.450 \mathrm{H} \\
/ 1.450 \mathrm{H} \\
166 \mathrm{~m}^{2} \text { efectiva } \\
525 \text { strokes } / \mathrm{m} \\
1-37 \mathrm{~kW} ; 2-55 \mathrm{~kW} \\
10 \\
7\end{array}$ \\
\hline $\begin{array}{l}\text { Horno rota- } \\
\text { tivo }\end{array}$ & $\begin{array}{l}\text { Tamaño } \\
\text { Pendiente } \\
\text { N. }{ }^{\circ} \text { de soportes } \\
\text { Velocidad } \\
\text { Accionamiento } \\
\text { Quemador }\end{array}$ & $\begin{array}{l}5,5 \mathrm{~m} \varnothing \times 100 \mathrm{~m} \mathrm{~L} \\
4 \% \\
4 \\
0,7 \simeq 3,5 \mathrm{r} / \mathrm{min} \\
2-400 \mathrm{~kW} \mathrm{c.} \mathrm{continua} \\
\text { Máx. } 14 \mathrm{t} / \mathrm{h}\end{array}$ & $\begin{array}{l}\text { Filtro para } \\
\text { el aire de } \\
v \text { e } n \dagger \text { te } \\
\text { enfriador }\end{array}$ & $\begin{array}{l}\text { Tipo } \\
\text { Capacidad } \\
\text { Temperatura } \\
\text { gas } \\
\text { Contenido } \\
\text { polvo }\end{array}$ & $\begin{array}{l}\text { Multiciclones + Electro- } \\
\text { filtro } \\
2-8.000 \mathrm{~m}^{3} / \mathrm{min} \\
250^{\circ} \mathrm{C} \\
\begin{array}{l}\text { Entrada } 10 \simeq 30 \text { gra- } \\
\text { mos } / \mathrm{Nm}^{3} \\
\text { Salida } 30 \text { miligra- } \\
\text { mos } / \mathrm{Nm}^{3}\end{array}\end{array}$ \\
\hline
\end{tabular}

Esta planta se terminó en diciembre de 1973 y lleva funcionando sin problemas cerca de tres años.

Alcanza las $8.000 \mathrm{t} /$ día de producción, con un consumo calorífico de 740 a $750 \mathrm{kcal} / \mathrm{kg}$ de clínker. En la tabla 2 se indican unos datos recientes relativos a las condiciones de operación del sistema de clinkerización I.H.I.-S.F.

En esta planta, cerca del $62 \%$ del total del fuel es quemado en el horno instantáneo de alta eficiencia (horno flash), produciéndose en el material una calcinación de aproximadamente el $88 \%$. Así pues, con un horno rotativo del mismo diámetro, con el sistema S.F. es doble que con un S.P. y cuatro veces la de un sistema húmedo.

Con el sistema S.F. la cantidad de aire de combustión, que en los sistemas convencionales es extraído del enfriador a través del horno, es aquí dividido en dos partes. La temperatura del aire secundario para el horno rotativo se encuentra por encima de los $1.100^{\circ} \mathrm{C}$, mientras que para el horno flash está entre $750^{\circ}$ y $780^{\circ} \mathrm{C}$. Es imprescindible para la operación del enfriador de clínker que el aire de combustión para el horno sea extraído con la mayor temperatura posible, mientras que el aire menos caliente es conducido al horno flash. A este respecto, lo más importante es el adecuado control del espesor de la capa de clínker y la proporción de flujo de aire de enfriamiento en la zona de recuperación del calor.

Del espesor de la capa de clínker debe resultar un rendimiento térmico general en el enfriador de aproximadamente un $70 \%$ y que los posibles daños en las placas de las parrillas sean evitados. 


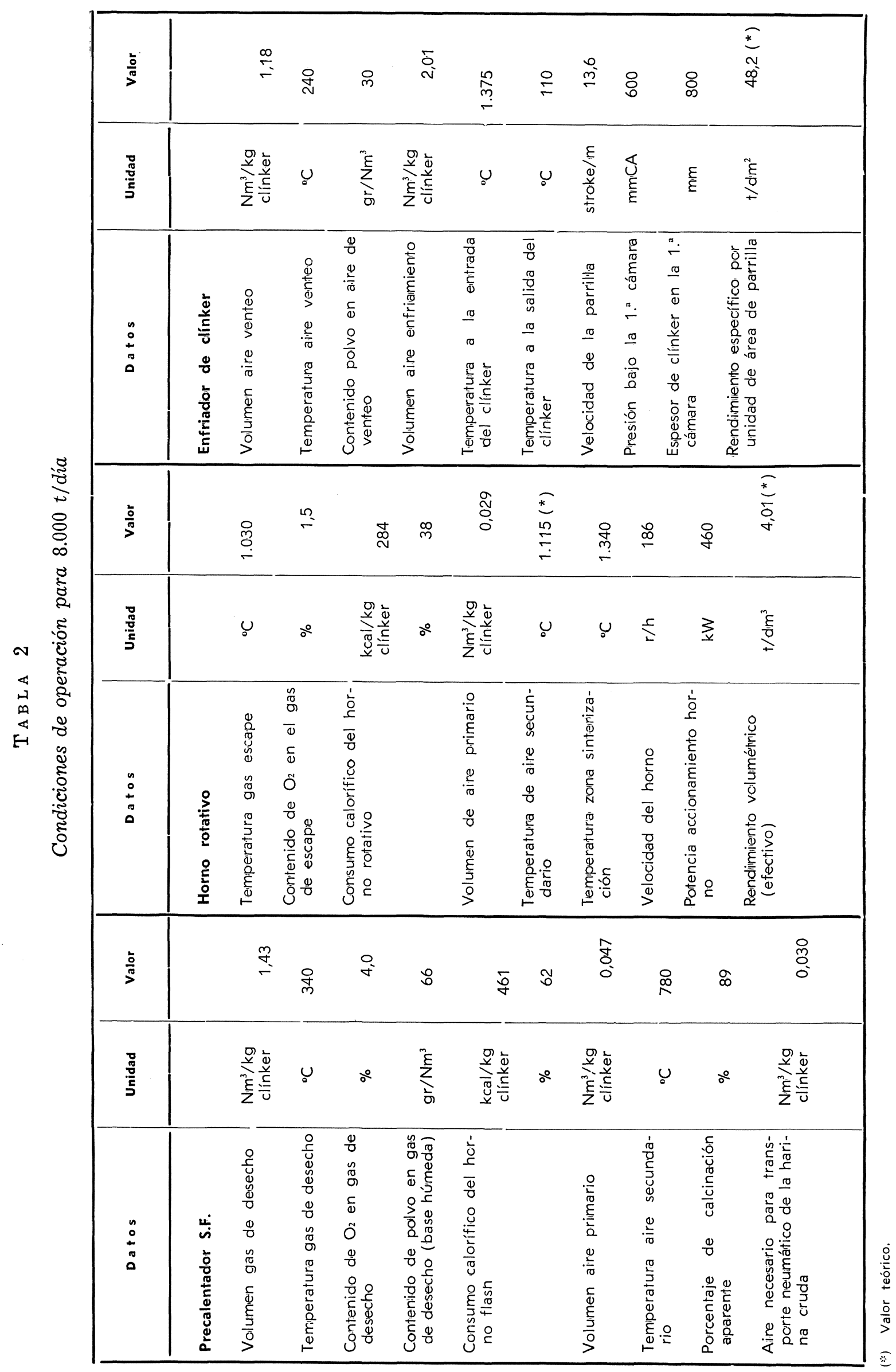


El aire de combustión para el horno flash, conducido a través del conducto de aire secundario, se mezcla con el gas del horno a $1.030^{\circ} \mathrm{C}$ en el conducto de subida al horno flash. El punto de mezcla se encuentra muy cerca de la boca del horno, estando diseñado de forma que la mezcla de gases sea perfecta e instantánea. Debido a esta ventajosa situación del precalentador S.F., el gas del horno conteniendo álcalis volátiles en fase gaseosa es enfriado por el aire secundario, como resultado de lo cual los álcalis se condensan en la superficie de las partículas de polvo que son arrastradas desde el horno. Como consecuencia, normalmente no suele ser necesario limpiar las paredes del precalentador y no hay peligro de atascamiento de ciclones. Los trabajos de limpieza, en caso de ser requeridos, se limitan a la zona ascendente desde la boca del horno y antes del punto de mezcla.

Es esencial para mantener estable la operación del proceso, conservar constante el porcentaje de calcinación en el material alimentado al horno rotativo. No obstante, debido a las variaciones de contenido de $\mathrm{CO}_{3} \mathrm{Ca}$ en la harina y a otras causas, se producen fluctuaciones de poca importancia en el mencionado porcentaje, aunque el fuel en el horno flash sea mantenido constante. Teniendo en cuenta que la calcinación de la harina cruda es principalmente gobernada por la temperatura de la reacción, la proporción de fuel para el horno flash es controlada automáticamente para que se mantenga constante la temperatura del gas que sale del cuarto ciclón (ver fig. 9). Por este motivo, en la planta mencionada las variaciones en la calcinación en la boca del horno, medidas actualmente, están siempre entre $\pm 2 \%$. Además, el tanto por ciento de precalcinación en el S.F. puede cambiarse a voluntad, ajustando en el cuadro de control la temperatura. De este modo, es posible optimizar todo el proceso de operación.

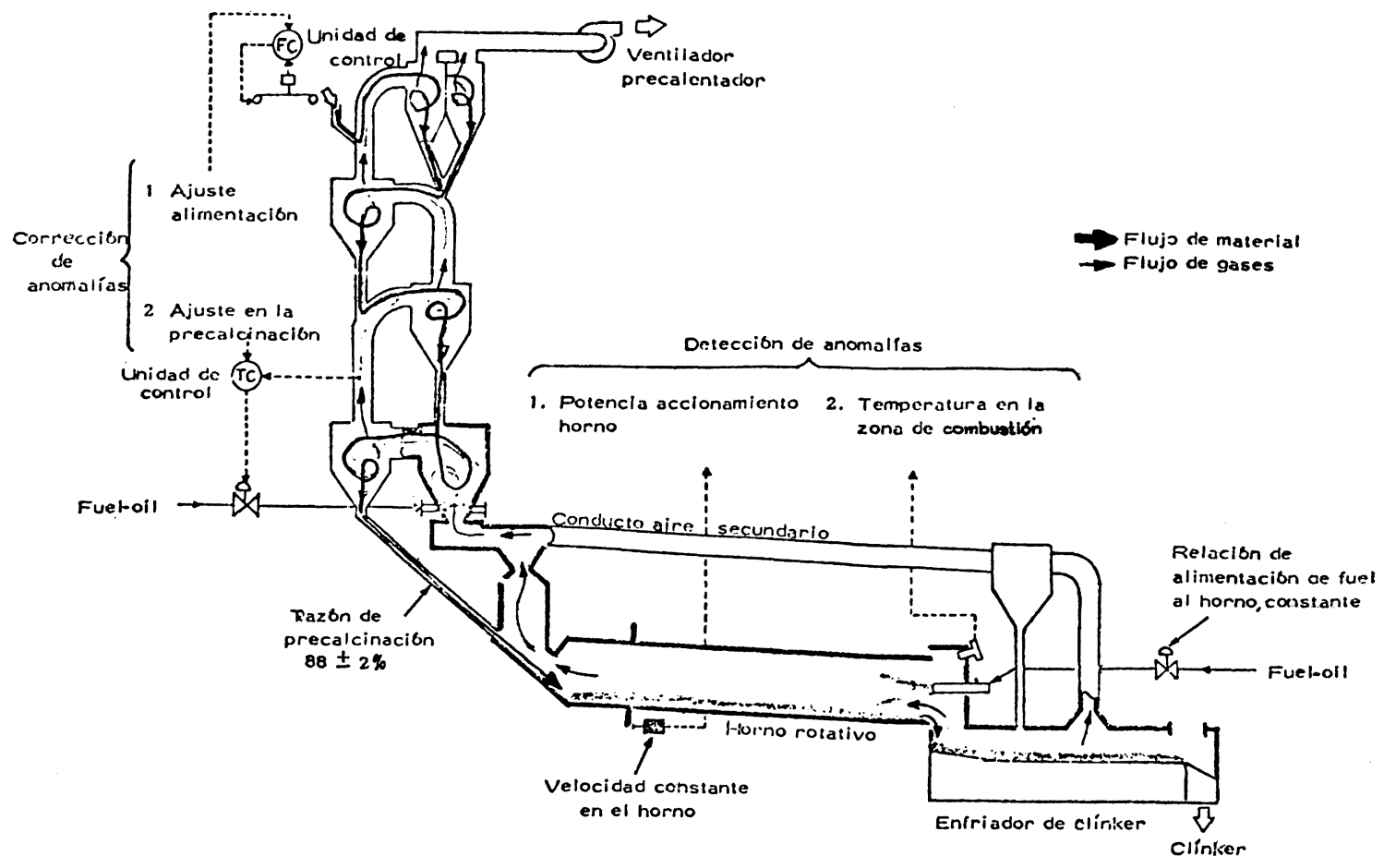

Fig. 9.-Control de precalcinación y condiciones de cocción.

Como consecuencia del ya mencionado control en el precalentador de la precalcinación, la distribución de temperaturas en el horno rotativo es así mismo mantenida constante, mientras que el flujo de harina, el cual es observado ocasionalmente con un sistema convencional S.P., nunca ha sido observado en este sistema. 
En operación normal, la proporción del fuel y la velocidad de giro del horno son mantenidas constantes, por lo que las condiciones de clinkerización son finalmente ajustadas por el rendimiento y la proporción de precalcinación.

$\mathrm{Si}$ ocurriese alguna anomalía repentina, por causa de pegaduras en las paredes del horno, por ejemplo, puede ser detectada enseguida por variaciones en la potencia de accionamiento del horno, más exacto que en cualquier otro proceso, ya que la mayor parte del horno está ocupada por la zona de sinterización. Los cambios en la potencia de accionamiento anticipan cambios en la temperatura de la zona de quemado, facilitando así el control del horno.

En el caso de que la anomalía no sea grave, pueden ser mantenidas las condiciones de funcionamiento normales, simplemente reduciendo la proporción de harina cruda en la alimentación en un pequeño porcentaje, durante 20 ó 30 minutos. Aun en caso de que ocurra una anomalía grave, la cual se ha producido con escasa frecuencia, la unidad de control de temperatura en el gas de salida del cuarto ciclón es incrementado en $5^{\circ} \mathrm{C}$, además del anteriormente mencionado control sobre la proporción en la alimentación de harina; por lo que, en la combinación de ambos efectos, de una parte el incremento de la proporción en la calcinación y de otra el descenso de carga en el horno, desembocará en la corrección de la anomalía en menos de una hora.

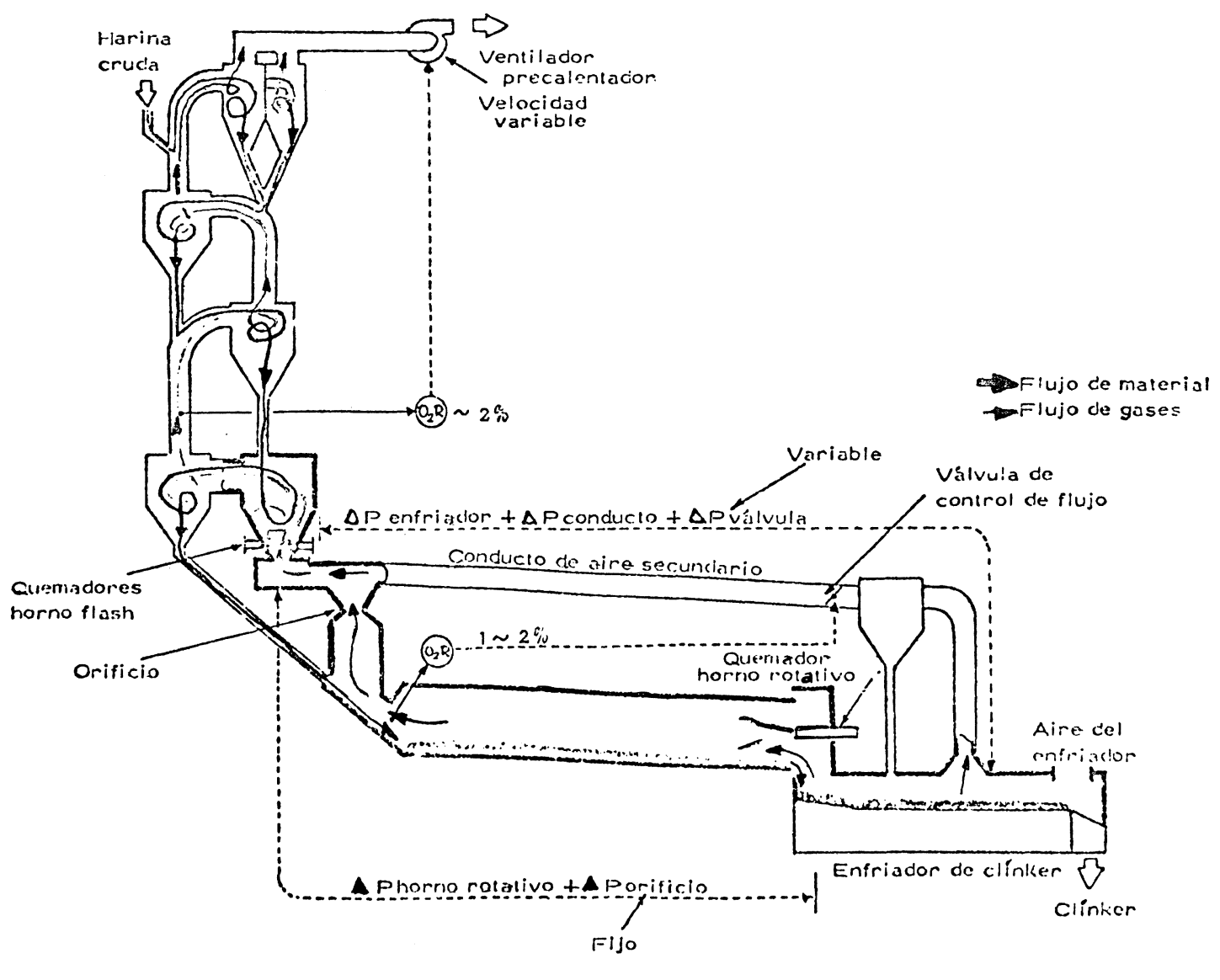

Fig. 10.-Esquema de control del sistema S.F.

Es importante, para optimizar la operación del horno, así como para la economía térmica del proceso, que la combustión de fuel sea efectuada con el mínimo gasto de aire. La explicación de cómo se realiza el control de la combustión en el sistema S.F. se puede ver en la figura 10. El horno flash se construye entonces de forma que no requiera para el aire de combustión secundario una alta energía cinética. De esta manera, no es ne- 
cesario colocar en el conducto de aire secundario ningún tipo de ventilador, que sufriría los efectos del abrasivo polvo de clínker, a la vez que limitaría la temperatura del aire caliente recuperado del enfriador de clínker. En el sistema I.H.I.-S.F., se instala un pasaje $\mathrm{u}$ orificio fijo en la zona alta de paso de gases del horno al precalentador para realizar una ligera disminución en la resistencia de tiro del horno respecto al conducto de aire secundario. Además, se construye en el conducto de aire secundario una válvula de control de flujo, lo que permite regular el aire de combustión para el quemador del horno rotativo y los quemadores del horno flash independientemente. Por otra parte, la velocidad del ventilador de tiro inducido del precalentador también se regula, con el fin de asegurar que el contenido en oxígeno a la salida de los gases del cuarto ciclón sea alrededor del $2 \%$. Así pues, las relaciones de aire en exceso para las dos combustiones pueden siempre mantenerse en las condiciones óptimas.

\section{E N T A J A S}

Una de las ventajas más destacables del sistema S.F. es la duración de los ladrillos refractarios. Por el aumento de la capacidad unitaria en el sistema S.P., la duración de los ladrillos refractarios se acorta debido al incremento de la carga térmica por sección del horno y por la deformación de la envolvente del horno; en consecuencia, los trabajos de renovación se dificultan, aparte la relación tiempo-consumo. Por este motivo no resulta económico construir grandes hornos S.P. a la vista de los costes de mantenimiento y disponibilidad de los mismos.

En el sistema S.F., el rendimiento del horno resulta dos veces el de un S.P. Pero, además, más del $60 \%$ del fuel que se consume es quemado en el horno flash, lo que supone disminuir la carga térmica del horno a menos del $80 \%$ respecto a un horno tipo S.P. En resumen, al ser el funcionamiento del horno absolutamente estable y existir pequeños choques térmicos y químicos, la duración del refractario en un horno S.F. es más larga que la de un horno S.P. del mismo tamaño, el cual, por otro lado, sólo obtiene la mitad de producción.

La figura 11 muestra una relación de valores sobre el consumo específico de ladrillos básicos referidos a la capacidad unitaria de hornos S.P., basada en un informe del Comité del Fuel de la Asociación de la Industria del Cemento de Japón realizado en el año 1972.

La curva que figura en el gráfico referente al sistema S.F. se ha realizado representando varios consumos de hornos S.F. en funcionamiento, uniéndolos por una línea. La línea es casi plana y la media del consumo está alrededor de $\operatorname{los} 0,2 \mathrm{~kg} / \mathrm{t}$ de clínker que representa de un tercio a un quinto de lo que requeriría un horno S.P. Como ejemplo se puede decir que el ahorro anual en ladrillos refractarios para una planta de $3.000 \mathrm{t} /$ día de producción de clínker se acerca a la cantidad de 22.400 .000 pesetas, tomando como precio por tonelada de ladrillo básico, ya colocado, el de 37.400 pesetas.

Vamos ahora a señalar algunas de las ventajas de una planta de producción de clínker de cemento por el sistema S.F. frente a una S.P.

\section{Capacidad}

En comparación con el sistema S.P. convencional, el rendimiento es doble. Tres plantas con una producción de 7.000 a 8.000 t/día han sido realizadas, siendo el tamaño del horno compatible técnicamente. 
Todo esto ayuda a reducir en gran medida costos de personal por unidad de producción.

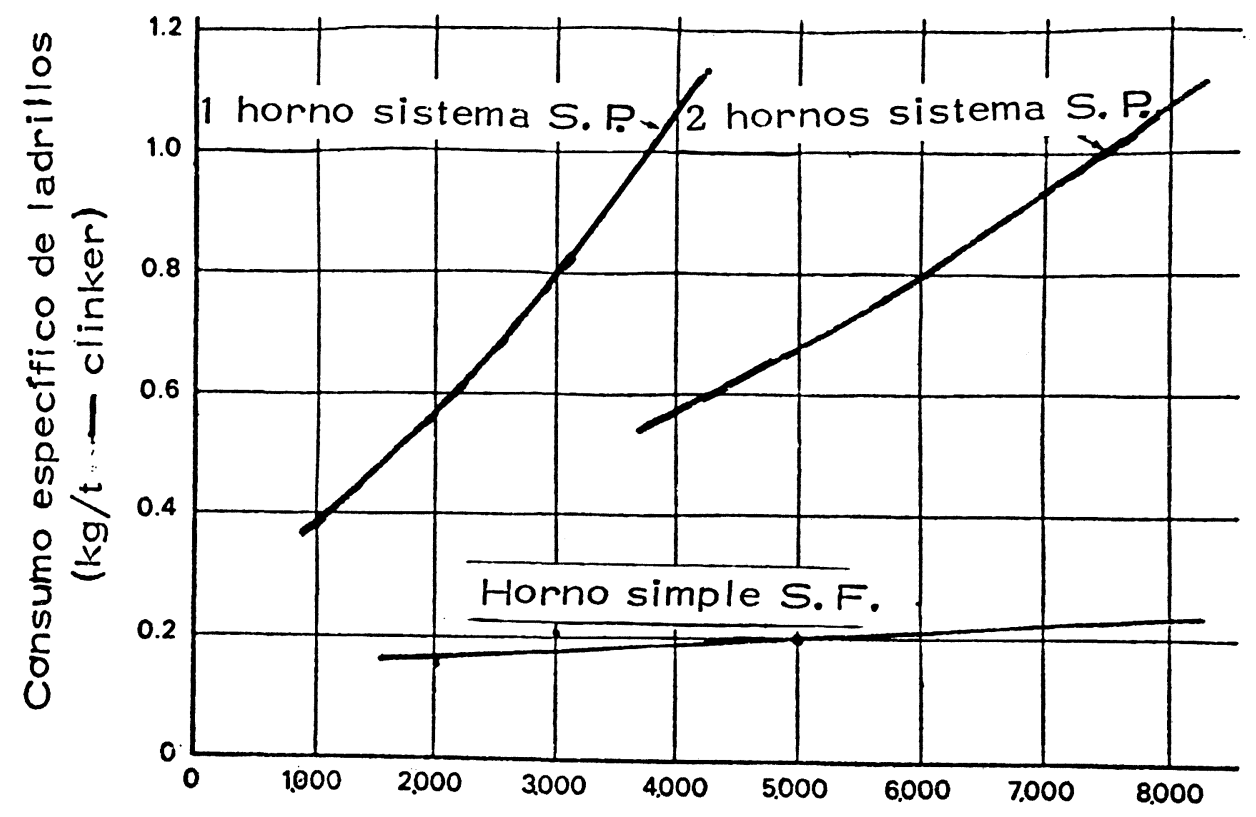

\section{Capacidad ( $t / d)$}

Fig. 11.-Consumo específico de ladriltos básicos para hornos con sistemas S.P. y S.F.

\section{Costos de instalación}

El ahorro en el costo inicial del sistema S. F. frente al S.P. será mayor debido al incremento de capacidad. Supondrá un $10 \%$ en una planta de 3.000 t/día y más del $20 \%$ en una de 8.000. Por supuesto, para conseguir esta última producción en un sistema convencional (S.P.), ha de dividirse en dos hornos rotativos, dos precalentadores y dos sistemas de enfriamiento de clínker.

\section{Area de instalación}

La superficie necesaria para instalar un S.F. se reduce, frente a los convencionales en un $25 \%$.

\section{Consumo de ladrillos en el horno}

Tal como se ha dicho anteriormente, el consumo de ladrillos refractarios en un horno S.F. oscila entre un tercio y un quinto del de un horno S.P.

\section{Producción anual}

Contar con una vida más larga del refractario permite reducir los tiempos de parada para reparar de 10 a 20 días. Además, los hornos resultan más pequeños, pudiendo ser calentados en menor tiempo, con lo que un sistema S.F. puede alcanzar la capacidad normal en 18 horas sin dificultad. A causa de estas condiciones, la producción anual de un horno S.F. será de un 5 a un $8 \%$ más alta que para un horno S.P. 


\section{Sistema de operación}

Cerca del $90 \%$ de la calcinación en la harina cruda se realiza antes de que ésta entre en el horno rotativo, precisamente en el precalentador S.F., siendo minimizadas las fluctuaciones en la calcinación mediante control automático del fuel en el horno flash. Por ello, en condiciones normales de operación, no hay necesidad de variar la cantidad de fuel y la velocidad de giro del horno.

Según todo esto, el proceso S.F. no requiere operadores experimentados y, en conjunto, la operación resulta más fácil, siendo el clínker producido más uniforme que en los hornos S.P.

\section{Control por computador}

Como la transferencia de calor en el horno flash es realizada instantáneamente y el tiempo de retención en el horno es de sólo 20 minutos, el control por computador puede ser aplicado de la manera más racional.

\section{Polución por óxido de nitrógeno}

Debido a que la combustión en el horno flash tiene lugar con un bajo contenido de mezcla de aire de combustión y gas del horno y a temperatura inferior a los $900^{\circ} \mathrm{C}$, no sólo no se producirán los óxidos de nitrógeno del fuel suministrado al horno flash, sino que los óxidos de nitrógeno contenidos en el gas del horno son reducidos a la mitad. De hecho la concentración media de óxido de nitrógeno resulta ser solamente de una tercera a una quinta parte de la existente en los sistemas convencionales.

\section{By-pass}

Con el propósito de reducir el contenido de álcalis en el clínker o prevenir las posibles anomalías en el funcionamiento debidas a las pesadas costras, el sistema de by-pass para los gases del horno que se viene aplicando en la industria puede ser usado para este sistema. Sin embargo, pueden obtenerse algunas ventajas frente al sistema convencional, ya que la concentración de álcalis en la corriente de gases de salida del horno es aproximadamente el doble. Por lo tanto, la cantidad de álcalis requerida puede ser eliminada a través del by-pass con la mitad del volumen de gases. Como resultado, la pérdida de calor en el gas del horno de desecho y los costos iniciales de inversión para equipos adicionales se pueden reducir a la mitad respecto a los sistemas S.P.

Después de completarse la primera planta S.F. en el año 1971 han sido vendidas veintidós más, de las cuales dieciséis están actualmente funcionando sin ningún problema.

El primer horno S.F. en Europa es de 4.000 t/día y ha sido puesto en marcha recientemente en Grecia. Los datos recogidos a través de la operación de todas estas plantas S.F. han sido aplicados con el fin de conseguir la optimización en los criterios de diseño, así como para lograr que las condiciones de operación sean más y más estables. Ahora que los proyectos del sistema de cocción de clínker de cemento I.H.I.-S.F. son realizados en España por FELGUERA-I.H.I., S. A., bajo la asistencia técnica de I.H.I. con la cooperación de estas dos compañías, es de esperar que el sistema S.F. sea ampliamente difundido y obtendrá una gran reputación en la industria española del cemento. 


\section{COLOQUIO}

1. Lo abrió el Presidente, Sr. PEREZ OLEA, y lo inició el Sr. CALLEJA planteando algunas cuestiones sobre álcalis. ¿Cómo se manifiestan — preguntó-, los problemas de álcalis y adherencias en el sistema SF, en comparación con el sistema SP? ¿Hay diferencias de orden cualitativo y/o cuantitativo? ¿Cómo se resuelven las dificultades a que dichos problemas pudieran dar lugar?

El Sr. KOBAYASHI, a través del Sr. GARCIA RIERA, contestó que los problemas son prácticamente los mismos en ambos sistemas. Es decir, que aproximadamente del 95 al $98 \%$ de los álcalis van al clínker, y que cuando éste resulta muy alto en álcalis es preciso disponer de un by-pass de gases. Añadió que los gases del enfriador en el sistema SF se dividen en dos partes, enviándose una como aire secundario de combustión -a temperatura más baja - al horno "flash", y otra como aire secundario de combustión -a temperatura más alta- al horno rotatorio; y que esta segunda parte, que es de la que se elimina una porción por el by-pass, contiene prácticamente todos los álcalis, a una concentración aproximadamente doble de la que es normal en otro sistema, por lo cual la eliminación de una cierta cantidad de estos álcalis se consigue desviando por el bypass un volumen de gases también mitad del normal, con evitación de la consiguiente pérdida de calor.

2. A continuación el Sr. LONDOÑO preguntó acerca de la experiencia que pudiera haber en el empleo del carbón como combustible en el proceso SF, a lo que el Sr. KOBAYASHI respondió que por el momento no se tenía ninguna, ya que no se han montado fábricas del sistema SF que trabajen con carbón; pero que el año próximo se iba a utilizar carbón en una de ellas; y que la experimentación llevada a cabo hasta el momento ponía de relieve que no hay ninguna dificultad en quemar en el horno "flash" carbones bituminosos con $25 \%$ (de 20 a $30 \%$ ) de materias volátiles. A la pregunta del Sr. LONDOÑO de dónde estaba situada esa fábrica, el Sr. KOBAYASHI respondió que en el Este de los Estados Unidos.

3. Después el Sr. FRANCO deseó saber cuál era el tamaño mínimo de planta a partir del cual se justifica el empleo del sistema SF. A ello respondió el Sr. KOBAYASHI diciendo que había estado calculando el costo de la instalación SP comparado con el de $\mathrm{SF}$, y que se ve que en el margen de capacidades de producción entre las 1.500 y las: 2.000 t/día se sitúa el punto en que los costos de ambas instalaciones se igualan. Si se trata de fábricas mayores —añadió-, por ejemplo de 3.000 a 4.000 t/día, los costos específicos bajan en ambos casos y la diferencia entre el del sistema SP y el del sistema SF se hace mayor, en favor del SF.

4. En relación con el by-pass de gases el Sr. ESTRADA preguntó acto seguido cuántas kcal se estima que se pierden por cada $1 \%$ de gases eliminados del sistema por el bypass. En respuesta a ello el Sr. KOBAYASHI dijo que en el proceso SP el by-pass de un $1 \%$ de gases supone un incremento del 2 al 2,5\% en el consumo de calor, y que en el caso del proceso SF y en las mismas condiciones el incremento de dicho consumo es sólo del $0,5 \%$; o, expresado de otro modo, el aumento del consumo es en este último caso de $1 \mathrm{kcal} / \mathrm{kg}$ de clínker, por cada $1 \%$ de gas desviado por el by-pass. 
5. El Sr. CALLEJA volvió después sobre el tema del posible empleo del carbón en relación con las cenizas del mismo. Preguntó al Sr. KOBAYASHI si, de igual manera que al contestar a una pregunta anterior había dado valores para el contenido admisible $u$ óptimo de volátiles, podía dar una respuesta paralela en cuanto al contenido de cenizas. A ello respondió el Sr. KOBAYASHI que en el caso de las cenizas es preciso tener en cuenta su punto de fusión, y que en la medida en que se han efectuado ensayos con varios tipos de carbones bituminosos se ha visto que sus cenizas tienen un punto de fusión situado alrededor de los $1.300^{\circ} \mathrm{C}$ o superior, y que tales carbones con contenidos de cenizas del 15 al $20 \%$ no deben constituir problema alguno para ningún revestimiento de cualquier horno "flash".

6. Después intervino el Sr. SORIA acerca de los enfriadores, diciendo que, según el esquema de instalación que había visto, ésta iba provista de un enfriador de parrilla, del cual se recupera aire para la cámara "flash". Preguntó si se tenía alguna experiencia sobre enfriadores de satélites.

Le respondió el Sr. KOBAYASHI diciendo que, como había mostrado en los esquemas de las diapositivas, se necesita aire secundario para el horno "flash" que es conducido al mismo desde el enfriador, por lo cual no se puede emplear uno de tipo planetario, como en el sistema SP, sino que es preciso tenerlo de otro tipo; o bien de satélites, pero combinado con otro de tipo diferente. En este último caso -añadió-, el aire secundario del horno rotatorio debe proceder del enfriador de satélites, mientras que el aire de combustión del horno "flash" debe ser el del otro enfriador (por ejemplo de tipo rotatorio y montado a continuación del anterior), el cual es llevado a la cámara "flash" a través del conducto secundario. Existe, pues - dijo-, la posibilidad de hacer el enfriamiento en dos etapas: una de enfriador planetario, en la que el aire de enfriamiento iría al horno rotatorio, y otra de enfriador de otro tipo, por ejemplo tubular giratorio, cuyo aire de enfriamiento se llevaría al horno "flash" por el conducto secundario.

El Sr. SORIA puntualizó que tenía noticia de que en Europa hay firmas que estudian este sistema, precisamente para eludir el enfriador de parrilla, y que agradecería que alguien que pudiera hablara sobre ello, así como sobre la posibilidad de utilizar combustibles sólidos, ya que a su juicio eran dos aspectos en los que en Europa se estaba trabajando con interés.

7. A continuación preguntó el Sr. VARGAS si era rentable la transformación de un horno SP en SF. Según la traducción hecha de la respuesta del Sr. KOBAYASHI, la transformación de un horno al sistema SP en otro del sistema SF es siempre posible, y al hacerla se duplica - como ya quedó expuesto en la ponencia- la capacidad de producción. La transformación exige una serie de modificaciones, como es la de instalar un enfriador mayor para la nueva producción doble, y como es la de aumentar la velocidad de giro del horno prácticamente al doble, por la misma razón; por supuesto, habría que añadir la torre del precalentador. Y, desde el punto de vista de la rentabilidad -añadió el Sr. KOBAYASHI-, por descontado que la transformación de una planta SP que ya se tiene, en otra SF, es mucho más rentable que montar una SF nueva, pues a mucho menor costo se duplica la producción, como queda dicho.

8. En relación con el mismo tema el Sr. MARTINEZ YNZENGA no consideró del todo contestada la pregunta anterior, y quiso saber si en la transformación de un horno convencional a otro del sistema SF la economía es tal que realmente merece la pena tal transformación, en relación con el costo de una instalación SF nueva, y dado que la parte que se aprovecha de la instalación ya existente es realmente pequeña.

El Sr. KOBAYASHI respondió que, en efecto, del horno en sí se aprovecha sólo una parte, aunque no tan pequeña; pero que lo que se aprovecha en su totalidad es la infraestruc- 
tura resistente de la fábrica. Y añadió: al hablar de transformar un sistema SP, u otro cualquiera de vía seca o de vía húmeda, en un sistema $\mathrm{SF}$, hay que pensar que siempre se incrementa la producción en gran medida; entonces la rentabilidad del cambio va a depender más del cementero que sabe con qué capacidad de molienda de crudo y de clínker cuenta, y si ambas son capaces de absorber el aumento de producción que el cambio va a permitir. Si es así, es distinto que si el posible aumento de producción le obliga a cambiar molinos, silos y, en definitiva, la instalación. Otra consideración importante - prosiguió-, en el caso de que disponga de varios hornos, es la de si la trasnformación de uno o dos de ellos al sistema SF le va a permitir prescindir de alguno o de todos los restantes, manteniendo o incluso incrementando la producción con el cambio. En tal caso éste es mucho más rentable que montar el correspondiente sistema SF nuevo y completo. Los números - concluyó-, lo demuestran, pues en definitiva se trata de sustituir hornos de más bajo rendimiento por uno solo de rendimiento mejor y capaz de dar por si sólo la producción de toda la instalación preexistente.

Y no habiendo más preguntas, el Presidente Sr. PEREZ OLEA agradeció una vez más la actuación de todos los participantes en el Coloquio y lo dió por concluido. 